\title{
Valores como Preditores da Satisfação com a Vida em Jovens
}

\author{
Cátia Marques - Universidade do Minho, Braga, Portugal \\ Ana Daniela Silva - Universidade do Minho, Braga, Portugal \\ Maria do Céu Taveira - Universidade do Minho, Braga, Portugal
}

\begin{abstract}
Resumo
Numa perspectiva funcionalista, os valores são uma expressão cognitiva das necessidades humanas, princípios que orientam o comportamento para metas mais pessoais, sociais ou gerais de vida, cujo impacto psicossocial merece mais investigação. Neste estudo, analisou-se em que medida o tipo de orientação dos valores prediz a satisfação com a vida de 562 jovens portugueses ( $n=303,53,9 \%$ mulheres), com idades entre 14 e 22 anos $(M=16,9, D P=1,71)$, que frequentam o ensino médio: via profissionalizante $(63,7 \%)$ e regular (36,3\%). Os jovens completaram o Questionário de Valores Básicos e a Escala de Satisfação com a Vida. Os resultados da análise de regressão linear indicam que a orientação dos valores explica menos de um quarto da variabilidade da satisfação com a vida, o que suscita a necessidade de se analisar o seu poder explicativo em conjunto com outras variáveis, por exemplo, de personalidade e sociocognitivas.
\end{abstract}

Palavras-chave: valores, satisfação, predição, jovens

Values as predictors of life satisfaction in youths

\begin{abstract}
In a functionalist perspective, values are a cognitive expression of human needs as well as principles that guide behaviors to personal, social or general life goals, whose psychosocial impact deserves more investigation. This study aimed to analyze whether the type of values orientation predicts life satisfaction in 562 Portuguese youths $(\mathrm{n}=303,53.9 \%$ women) aged 14 to 22 years $(M=16.9 ; S D=1.71)$, attending high school studies, being $63.7 \%$ technical and $(36.3 \%)$ regular high school. Students completed the Basic Values Survey and the Satisfaction With Life Scale. The results of the linear regression analyzes indicate that the values orientation explains less than a quarter of life satisfaction variability, which indicated the need to further analyze its explanatory power together with other variables, such as, personality and socio-cognitive.. Keywords: values; satisfaction; prediction; youth
\end{abstract}

Valores como predictores de satisfacción con la vida en los jóvenes

\begin{abstract}
Resumen
Desde el punto de vista funcional, los valores son una expresión cognitiva de las necesidades humanas, principios que orientan el comportamiento hacia metas más personales, sociales o generales de vida, cuyo impacto psicosocial necesita más investigación. En este estudio se analizó en qué medida el tipo de orientación de los valores predice la satisfacción con la vida de 562 jóvenes portugueses ( $\mathrm{n}=303,53.9 \%$ mujeres), con edades entre 14 y 22 años $(M=16.9, D P=1.71)$ que frecuentan Enseñanza Secundaria: profesional (63.7\%) y normal (36.3\%). Los jóvenes completaron el Cuestionario de Valores Básicos y la Escala de Satisfacción con la Vida. Los resultados del análisis de regresión lineal indican que la orientación de los valores explica menos de una cuarta parte de la variabilidad de satisfacción con la vida, lo que plantea la necesidad de analizar su poder explicativo, junto con otras variables, por ejemplo, de personalidad y socio-cognitivas.

Palabras-clave: Valores; satisfacción; predicción; jóvenes
\end{abstract}

Os valores são uma dimensão importante da construção do self durante a adolescência e juventude, com impacto significativo nas percepções, objetivos, atitudes e comportamentos dos jovens, ao nível da saúde, das relações interpessoais e sociais e da carreira (e.g., Bardi \& Goodwin, 2011; Brewer \& Roccas, 2001; Gouveia et al., 2008; Porfeli, 2007; Rokeach, 1973). Na investigação sobre os valores, a perspectiva funcionalista (Gouveia, 2003) oferece uma visão compreensiva do constructo, integrando, por exemplo, uma perspectiva mais individual (e.g., Rokeach, 1968, 1973; Schwartz, 1992), e uma perspectiva mais cultural (Hofstedt, 1984; Inglehart, 1977) sobre os valores. Constitui, por isso, um modelo útil e parcimonioso para estudar os valores básicos de vida em jovens.

Segundo a teoria funcionalista (Gouveia, 2003; Gouveia et al., 2008; Gouveia et al., 2010; Gouveia, Milfont, Fisher, \& Coelho, 2009; Gouveia, Milfont, \& Guerra, 2014), os valores são princípios que orientam a vida dos indivíduos e uma expressão cognitiva das necessidades humanas. Isto é, são motivadores da ação e, por isso, podem ser organizados ao redor de duas funções ou vectores principais: o tipo de orientação mais pessoal, central ou social; e o tipo de motivação - mais materialista ou idealista. Cada uma das seis subfunções resultantes do cruzamento desses dois vetores 
caracteriza-se por três valores específicos. Assim, o tipo de orientação pessoal inclui valores ligados às subfunções de experimentação (emoção, prazer e sexualidade) e de realização (poder, prestígio e sucesso). A orientação central inclui valores de natureza suprapessoal (beleza, conhecimento, maturidade) ou relacionados com a existência (saúde, estabilidade pessoal, sobrevivência). A orientação social inclui valores de natureza mais interativa (afetividade, apoio social, convivência) e normativa (obediência, religiosidade, tradição). No segundo vetor, os valores pessoais de experimentação, centrais de tipo suprapessoal, e sociais de tipo interativo, definem a motivação idealista, enquanto os valores pessoais de realização, centrais de existência e sociais normativos, definem a motivação materialista.

Apesar da literatura sobre os valores evidenciar mais a sua estabilidade do que a sua mudança no tempo (e.g., Bardi \& Goodwin, 2011; Schwartz, 2005), existe evidência para pensar que a mudança nos valores pode ocorrer (e.g., Gouveia, Vione, Milfont, \& Fisher, 2015; Sheldon, 2005), refletindo-se nos valores que cada pessoa considera serem mais importantes para si em diferentes momentos da vida, e na hierarquia distinta dos valores em diferentes grupos da população (e.g., estudantes do ensino médio e universitários; indivíduos de diferentes regiões). Essas mudanças podem ocorrer fruto, por exemplo, de condições sociodemográficas, experiência e desenvolvimento pessoal, acontecimentos sociais, cultura ou processos de socialização. Quer os estudos transversais, quer sobretudo os estudos longitudinais sobre os valores básicos de vida, poderão esclarecer melhor essa problemática, dada a escassez da investigação sobre a estabilidade dos valores de vida (Bardi \& Goodwin, 2011).

Outra linha de estudo importante sobre os valores tem demonstrado o seu impacto a nível psicossocial. Por exemplo, no âmbito da perspectiva funcionalista, Vasconcelos (2004) e Pimentel (2004) verificaram que valores normativos relacionam-se negativamente com condutas antissociais em adolescentes e jovens. Outros estudos registram uma relação significativa entre os valores normativos, como a tradição, e a identidade social (e.g., Gouveia, Albuquerque, Clemente, \& Espinosa, 2002). Em um outro estudo sobre valores e sexismo, ficou demonstrado que as crenças e comportamentos de tipo conservador tendem a promover o sexismo, enquanto pessoas com crenças e comportamentos orientados para o universalismo (orientação central), tendem a ser menos sexistas (Belo, Gouveia, Raymundo, \& Marques, 2005). Pronk (2010) procurou conhecer em que medida as autoimagens e os valores pessoais e sociais de vida se relacionam com a imagem corporal em jovens. Os resultados desse estudo indicam que uma autoimagem independente e valores pessoais são preditores significativos do investimento corporal, o qual afeta a insatisfação com o corpo.

Por sua vez, Coelho, Gouveia e Milfont (2006), testaram o papel dos dez tipos de valores humanos de Schwartz (1992) em relação às atitudes ambientais, tendo concluído que os valores de autotranscendência, e especificamente os de orientação universalista (orientação central), predizem atitudes e comportamentos pró-ambientais. Gouveia et al. (2010) procuraram conhecer em que medida os valores básicos de vida explicam as metas de realização acadêmica. Os resultados das análises de correlação mostram que os valores se relacionaram mais com as metas de aproximação (aprendizagem e execução). A subfunção normativa foi preditora das metas de aprendizagem-evitação e de execução-evitação. As subfunções suprapessoal e existência predisseram a meta de aprendizagem-aproximação. E as subfunções de realização, normativa, interativa e suprapessoal predisseram a meta de execução-aproximação.

Em outro estudo, Gouveia et al. (2008) procuraram identificar em que medida os valores básicos de vida se relacionam com os interesses vocacionais dos adolescentes. Os resultados demonstraram que os valores básicos de vida e os interesses vocacionais estão correlacionados, sendo que pontuações elevadas em valores interativos, normativos e suprapessoais, correlacionaram-se positivamente com interesses vocacionais de tipo social. Registraram-se ainda correlações positivas entre valores de experimentação e de realização e interesses empreendedores, e negativas, entre valores suprapessoais e interesses empreendedores. Finalmente, os valores suprapessoais apresentaram correlação positiva com interesses de tipo realista (e.g., prático, mecânico, ar livre) e de tipo artístico.

Mais recentemente tem sido estudada também a relação entre valores numa perspectiva funcionalista e a satisfação com a vida (e.g., Albuquerque, Noriega, Coelho, Neves, \& Martins, 2006; Chaves, 2003; Fonseca, Chaves, \& Gouveia, 2006; Gusmão, 2004). O estudo das relações entre os valores básicos de vida e a satisfação com a vida pode permitir compreender melhor o papel dos valores no bem-estar pessoal e social (e.g., Brown \& Lent, 2016; Huta \& Waterman, 2014; Nogueira, 2015; Ramos, 2015). Com efeito, a satisfação constitui a dimensão mais cognitiva do bem-estar 
psicológico e relaciona-se com aspectos racionais ou intelectuais que o indivíduo experimenta, podendo avaliar-se de forma global, como satisfação com a vida, ou em termos específicos, como satisfação acadêmica, profissional ou conjugal (e.g., Diener \& Biswas, 2000; Diener, Suh, Lucas, \& Smith, 1999). Entre os antecedentes da satisfação com a vida, contam-se os traços de personalidade, a inteligência emocional, a satisfação em domínios específicos da vida, as relações pessoais e sociais, estruturas e sistemas organizacionais e de trabalho, e os sistemas social, cultural e econômico mais global (Brown \& Lent, 2016; Higgs \& Dulewicz, 2014).

Com base na perspectiva funcionalista dos valores, Chaves (2003) estudou uma amostra de adultos brasileiros e observou que os valores sociais de convivência e afetividade (subfunção interativa), e os valores centrais de maturidade (subfunção suprapessoal) e saúde (subfunção existência), foram os que mais predisseram a satisfação com a vida. Esses resultados indicam que a procura e eventual concretização de necessidades relacionadas com o crescimento pessoal no sentido da autorrealização, a segurança e o bem-estar físico e psicológico, os relacionamentos próximos e os afetos, ou o pertencimento a um grupo, apresentam maior relação significativa com a satisfação com a vida. Fonseca, Chaves e Gouveia (2006) estudaram outra amostra de adultos brasileiros, professores do ensino fundamental, e concluíram que os valores sociais normativos (obediência, religiosidade, tradição) apresentam uma correlação direta com a satisfação com a vida.

Gusmão (2004) estudou uma amostra de estudantes universitários brasileiros e verificou que apenas os valores sociais normativos e os valores pessoais de experimentação (emoção, prazer e sexualidade) explicaram a satisfação com a vida. Albuquerque Noriega, Coelho, Neves e Martins (2006) analisaram os resultados de uma outra amostra de jovens universitários e verificaram que os valores de orientação social e pessoal predisseram igualmente a satisfação com a vida, ainda que moderadamente, e que os valores de orientação central (e.g., beleza, conhecimento, saúde, sobrevivência) não predisseram a satisfação com a vida.

O conjunto de resultados com amostras brasileiras apresentado indica que determinados critérios, subfunções e valores básicos de vida estabelecem relações mais significativas do que outros com a satisfação com a vida. Contudo, o padrão de resultados é pouco consistente, sugerindo a necessidade de novos desenvolvimentos, como a replicação de estudos que analisem a relação entre os dois construtos em novas amostras.
Essa linha de estudo é importante, quer para o aprofundamento da relação entre valores básicos e satisfação com a vida, quer para a compreensão do papel dos valores, enquanto dimensão da personalidade. Nesse sentido, é objetivo deste trabalho prosseguir essa linha de investigação mais específica, analisando em que medida os valores básicos de vida são preditores da satisfação com a vida em jovens portugueses.

\section{Método}

\section{Hipóteses}

Tendo em conta os contributos apresentados sobre os valores básicos e a satifação com a vida, formularam-se as seguintes hipóteses de estudo:

Hipótese 1 - Os critérios valorativos (pessoal, central e social) predizem a satisfação com a vida.

Hipótese 2 - As subfunções valorativas (interativa, normativa, suprapessoal, existência, experimentação e realização) predizem a satisfação com a vida.

\section{Participantes}

A amostra deste estudo é constituída por 562 jovens, 303 mulheres $(53,9 \%)$ e 259 homens (46,1\%), com idades compreendidas entre os 14 e 22 anos de idade $(M=16,9, D P=1,71)$, tratando-se de uma amostra de conveniência. A maioria dos participantes frequentam o ensino profissionalizante/técnico $(63,7 \%)$ e os restantes frequentam o ensino regular $(36,3 \%)$. Os participantes são provenientes da região norte $(79,5 \%)$, centro $(4,8 \%)$ e ilhas $(15,3 \%)$ de Portugal.

\section{Instrumentos}

Para medir os valores básicos de vida, utilizou-se o Questionário dos Valores Básicos (QVB; Gouveia, et al., 2009). O QVB é composto por um total de 18 itens. Em cada item são apresentados dois exemplos, com o conteúdo de cada valor (e.g., "Exxito: obter o que se propõe; ser eficiente em tudo o que faz"). $\mathrm{O}$ indivíduo teve que se posicionar relativamente à importância do valor na sua vida, usando uma escala do tipo Likert em que 1 significa Totalmente não importante e 7 significa Extremamente importante. Verificou-se evidências de validade fatorial e consistência interna desse instrumento, em contexto brasileiro (Gouveia, 2003; Gouveia et al., 2009) e português (Marques, Silva, Taveira, Mota \& Gouveia, 2012). Além desses países, o estudo de Medeiros (2011) mostrou existir igualmente evidência empírica da adequação do instrumento em outros 11 países. Nesta amostra, os coeficientes de alfa variaram 
entre 0,45 (subfunção suprapessoal) e 0,59 (subfunção existência) e entre 0,64 (metas sociais) e 0,70 (metas centrais).

Para medir a satisfação com a vida, utlizou-se a versão portuguesa da Escala de Satisfação com a Vida (ESCV; Lent, Taveira, Sheu, \& Singley, 2009; Neto, 1993). Essa escala é composta por cinco itens, destinados a avaliar os juízos cognitivos globais de satisfação com a vida do indivíduo (e.g., "Na maioria dos aspectos, a minha vida está perto do meu ideal"). Foi pedido aos indivíduos que indicassem o grau de acordo e desacordo com cada um dos cinco itens, usando a escala dotipo Likert de 7 pontos, em que 1 significa Discordo totalmente e 7 significa Concordo totalmente. A ESV apresentou evidência empírica de validade fatorial e consistência interna em diferentes contextos, nomeadamente Portugal (Lent et al., 2009; Neto 1993, 2001; Neto, Barros, \& Barros, 1990; Simões 1992). Nesta amostra, o coeficiente alfa de Cronbach $(\alpha)$ foi de 83,1.

\section{Procedimento}

Os jovens foram convidados a participar no estudo voluntariamente e informados acerca do objetivo dele. Foi assegurado o sigilo das respostas, tratadas coletiva e estatisticamente. As respostas foram tratadas de modo confidencial, sem identificação dos respondentes, e avaliadas por especialistas de Psicologia creditados. À data da realização do estudo ainda não havia um comité ético estabelecido na instituição responsável pela investigação. No entanto, foram garantidos todos os cuidados éticos recomendados para esse tipo de estudos (e.g., consentimento informado, privacidade e confidencialidade) (Regulamento N 258/2011, Código Deontológico da Ordem dos Psicólogos Portugueses).

A coleta de dados aconteceu em um único dia. Os instrumentos foram administrados coletivamente em sala de aula por duas psicólogas, sendo enfatizado que as respostas deveriam ser dadas individualmente. Em média, foi necessário cerca de 15 minutos para concluir o preenchimento dos questionários. A ordem de aplicação dos instrumentos foi randomizada.

Para o processamento e análise de dados, foi utilizado o software Statistical Package for the Social Sciences - SPSS 21.0. Inicialmente, foram testados os pressupostos da análise de regressão linear. Posteriormente, procedeu-se à análise descritiva, cuja finalidade visa caracterizar o perfil da amostra. Seguiu-se a análise de regressão linear, com o método enter. Esta análise teve como intuito averiguar em que medida os tipos de orientação pessoal, central e social e as subfunções dos valores predizem a satisfação com a vida dos jovens.

Relativamente aos pressupostos seguiram-se as orientações de Stevens (1996) quanto à proporção de 15 observações por variável, para produzir estimativas confiáveis. Procurou-se também verificar o cumprimento do pressuposto de independência das observações, por meio do valor de Durbin Watson (Tabachnick \& Fidell, 2007). Quanto ao pressuposto de ausência de singularidade e de multicolinearidade, garante-se que não se incluem variáveis que são combinações lineares de outras já inseridas na análise; as correlações entre os preditores não foram superiores a 0,90 , valores de VIF inferiores a 4 e de Tolerance superiores a 0,1 (Tabachnick \& Fidell, 2007) foram tidos como critérios, indicando o cumprimento do pressuposto de multicolinearidade. O pressuposto de ausência de outliers foi verificado por meio do cumprimento do intervalo -3 e 3 de Standardized Residuals, bem como o máximo de 1 no Cook's Distance (Tabachnick \& Fidell, 2007).

\section{Resultados}

Os resultados das análises descritivas dos tipos de orientação pessoal, central e social, indicam que os jovens pontuaram mais nos valores centrais $(M=11,55$; $D P=1,78)$, seguido do pessoal $(M=10,62 ; D P=1,88)$ e, por último, do social $(M=10,16 ; D P=1,96)$. Os resultados da análise de regressão linear relativamente aos três tipos de orientação podem ser observados na Tabela 1.

Os resultados obtidos com a análise de regressão linear mostram que os tipos de orientação pessoal, central ou social dos valores básicos de vida explicam a satisfação com a vida, sendo que a orientação social é o preditor que mais explica a satisfação com a vida $\beta$ $=0,21, t=5,23, p<0,001$. Os indivíduos com valores mais elevados nesse tipo de orientação apresentam pontuações mais elevadas de satisfação com a vida. O modelo de regressão linear simples explica 4,5\% da variância $\left(R^{2}\right.$ Ajustado $\left.=0,04\right)$, sendo significativo, $F(1,575)=27,22, p<0,001$. Os resultados da análise de regressão linear relativa às subfunções valorativas podem ser observados na Tabela 2 .

Como se pode observar na Tabela 2, os resultados obtidos com a análise de regressão linear mostram que todas as subfunções valorativas explicam a satisfação com a vida, sendo que a experimentação revela ser um preditor significativo da satisfação com a vida percebida pelos jovens. Os indivíduos com valores mais elevados 
Tabela 1

Valores Sociais, Centrais e Pessoais como Preditores da Satisfação com a Vida

\begin{tabular}{lcccc}
\hline Valores & $\mathrm{R}^{2}\left(\mathrm{R}^{2}\right.$ Adjustado $)$ & $F(1,575)$ & $\beta$ & $t$ \\
\hline Social & $0,045(0,044)$ & $27,22^{* * *}$ & 0,21 & $5,23^{* * *}$ \\
Central & $0,024(0,022)$ & $14,14^{* * *}$ & 0,16 & $3,76^{* * *}$ \\
Pessoal & $0,037(0,035)$ & $21,98^{* * *}$ & 0,19 & $4,69^{* * *}$ \\
\hline
\end{tabular}

$* * * p<0,001$

Tabela 2

Subfunções Valorativas como Preditores da Satisfação com a Vida

\begin{tabular}{lcccc}
\hline Subfunção Valorativa & $\mathrm{R}^{2}\left(\mathrm{R}^{2}\right.$ Adjustado $)$ & $F(1,575)$ & $\beta$ & $t$ \\
\hline Experimentação & $0,039(0,03)$ & $20,63^{* * *}$ & 0,19 & $4,54^{* * *}$ \\
Realização & $0,026(0,02)$ & $14,07^{* * *}$ & 0,16 & $3,8^{* * *}$ \\
Suprapessoais & $0,020(0,02)$ & $10,03^{* * *}$ & 0,14 & $3,2^{* * *}$ \\
Existência & $0,019(0,02)$ & $9,63^{* * *}$ & 0,13 & $3,1^{* * *}$ \\
Interativa & $0,039(0,02)$ & $17,23^{* * *}$ & 0,17 & $4,2^{* * *}$ \\
Normativa & $0,035(0,04)$ & $22,05^{* * *}$ & 0,19 & $4,7^{* * *}$ \\
\hline
\end{tabular}

$* * * p<0,001$

na experimentação apresentam valores mais elevados da satisfação com a vida, $\beta=0,19, t=4,5, p<0,001$. O modelo de regressão linear explica $3,5 \%$ da variância $\left(R^{2}\right.$ Ajustado $\left.=0,03\right)$, sendo significativo, $F(1,575)=$ $20,63, p<0,001$.

\section{Discussão}

Este estudo teve como objetivo verificar se os valores básicos de vida predizem a satisfação com a vida de jovens portugueses. Adotou-se uma perspectiva funcionalista dos valores de vida, que os caracterizam a partir de três tipos de orientação-pessoal, central e social, com duas subfunções valorativas cada - experimentação e realização; suprapessoal e existência; interactiva e normativa, e dezoito valores singulares. Os resultados indicam que os valores básicos de vida predizem significativamente, ainda que com uma magnitude de efeito baixa, a satisfação com a vida. Ou seja, quanto mais priorizados são os critérios de tipo pessoal, central e social, maior é a satisfação com a vida. Os valores de orientação social são os que mais explicam a satisfação com a vida dos participantes do estudo. Tais valores incluem a afetividade, o apoio social e a convivência (subfunção interactiva), a obediência, a religiosidade e a tradição (subfunção normativa).
Para além disso, analisando o poder explicativo de cada uma das seis subfunções valorativas, verifica-se que é a subfunção de experimentação (valores de emoção, prazer e sexualidade), de motivação idealista, a que mais prediz a satisfação com a vida. Esses resultados sugerem que a valorização e eventual concretização de objetivos relacionados com a ligação afetiva, o suporte social e a interação com os outros, a adequação do comportamento às normas e tradições sociais, o desfrutar da vida e o prazer, estão entre os objetivos mais associados à satisfação com a vida, nos jovens estudados. Apesar de essa subfunção ser a que mais prediz a satisfação com a vida nessa amostra, a magnitude do efeito é ainda assim baixa. Como consequência, outras dimensões de personalidade (e.g., interesses, disposição afetiva, inteligência emocional), a satisfação em domínios da vida específicos (e.g., acadêmico, recreação, intimidade) e sociocognitivas (e.g., autoeficácia, expectativas de resultado), juntamente com os valores, podem explicar melhor a satisfação com a vida (Brown \& Lent, 2016; Higgs \& Dulewicz, 2014).

Os resultados apresentados são congruentes com resultados de estudos congêneres, em especial os reportados por Gusmão (2004), e Fonseca et al. (2006), e apoiam a hipótese de algum poder explicativo dos valores de orientação social e dos valores de 
orientação pessoal e motivação idealista (a experimentação), na satisfação com a vida. Além disso, indicam que os jovens portugueses estudados tendem a adotar um padrão valorativo central que engloba valores pessoais e sociais. Esse padrão pode justificar-se pela necessidade que cada indivíduo tem de procurar alcançar, na vida, tanto objetivos pessoais, como de interação com os grupos (Gouveia, 1998, 2003), e também pelo enfoque mais atual, no sistema educativo português, nas questões éticas e de cidadania associadas à aprendizagem escolar (e.g., Nogueira, 2015).

Trata-se também de um padrão de valores básicos de vida que tem registrado relações positivas com atitudes e comportamentos pró-sociais (e.g., antissexismo, pró-ambientais, metas de aproximação-execução na aprendizagem, identidade social) (e.g., Belo et al., 2005; Coelho et al., 2006; Gouveia et al., 2002; Pimentel, 2004; Vasconcelos, 2004) e interesses vocacionais de tipo social e empreendedor (Gouveia et al., 2008). À luz do que acontece em outras sociedades, os comportamentos pró-sociais e esse padrão de interesses vocacionais tem vindo a ser explicitamente muito valorizado nos ambientes educativos e profissionais portugueses, pelos pais, professores, empregadores, e restantes comunidades, dada a necessidade de formar trabalhadores com competências sociais e empreendedoras em todos os domínios e níveis da atividade laboral, para fazer face às constantes mudanças e desafios do mercado de emprego (Brown \& Lent, 2016).

Considera-se que este estudo contribuiu para o aprofundamento do estudo da satisfação com a vida, indicando que os valores básicos de vida estabelecem relações significativas com aquele construto. A partir dos resultados encontrados, pensa-se contribuir para o desenvolvimento de práticas de intervenção, tanto na escola, na família, como nas organizações, por meio da fomentação de valores em ambientes de referência, especialmente de tipo social, pois estes podem associarse à perceção de satisfação global com a vida.

Assim, considera-se que os resultados obtidos neste estudo trazem algumas implicações práticas no que diz respeito às intervenções sociais, no sentido de procurar uma melhoria na saúde sob uma perspectiva psicossocial. Evidencia-se a importância de compreender os mecanismos psicossociais envolvidos no processo de manutenção ou restabelecimento da satisfação com a vida. Concretamente, intervenções psicológicas mais específicas com jovens em contexto educativo, fomentando-se a importância de valores de caráter social, podem contribuir para promover vidas mais satisfeitas. Nesse sentido, pode ser útil recorrer à estratégia de criação de clubes comunitários ou de interesses sociais, nas escolas, para participação voluntária de alunos, docentes monitores e psicólogos coordenadores, onde os alunos possam desenvolver interesses e competências socioemocionais e resolver problemas concretos da comunidade, em grupo e com suporte social. Desse modo, poderá ser potencializada a satisfação com a vida dos alunos e, consequentemente, o seu maior envolvimento escolar e sucesso pessoal e vocacional.

Apesar dos contributos deste estudo, algumas limitações podem ser apresentadas, nomeadamente o fato da amostra se tratar de uma amostra de conveniência. Apesar da maior facilidade operacional e do baixo custo desse tipo de amostragem, esta tem como limitação a incapacidade de fazer afirmações gerais com rigor estatístico sobre a população.

Outra limitação do estudo é a não avaliação e inclusão na análise de outras variáveis que podem influenciar a satisfação com a vida. Poderiam ter sido incluídas no estudo, quer variáveis contextuais, quer psicológicas. A nível das variáveis contextuais, referiu-se, por exemplo, informação relativa ao estatuto socioeconômico da família dos participantes e até informação relativa à situação econômica e social do país à época da avaliação, uma vez que esses aspectos têm sido referidos na literatura como podendo ter impacto na satisfação com a vida (Ramos, 2015). Quanto a variáveis psicológicas, poderiam ter sido incluídas variáveis de personalidade (e.g., interesses, disposição afetiva). A consideração de outras variáveis poderia ter permitido aprofundar melhor o papel explicativo dos valores na satisfação global com a vida.

\section{Considerações Finais}

Este estudo permitiu perceber melhor como os valores básicos de vida de jovens estão associados à sua satisfação global com a vida e concluir que, apesar do seu pouco poder explicativo, os valores básicos de vida surgem associados à satisfação com a vida. Conclui-se que os valores de tipo pessoal, central e social predizem a satisfação com a vida, e que a subfunção experimentação é a que mais explica a satisfação, ainda que com pouco poder explicativo. Esses resultados levam a ponderar que outras variáveis de personalidade e sociocognitivas possam, juntamente com os valores, explicar a satisfação com a vida. Assim, considera-se que seria pertinente estudar a influência 
de outras variáveis nomeadamente de personalidade e sociocognitivas na explicação da satisfação com a vida. Além disso, pôde concluir-se que na amostra de jovens portugueses estudada, o padrão de valores central registrado, caracterizado por valores pessoais e sociais, indica a adoção de um padrão pró-social e construtivo de atitudes e comportamentos da parte dos jovens que pode ser favorecido e reforçado nos contextos educativos e de trabalho onde as crianças e os jovens se inserem.

\section{Referências}

Albuquerque, F., Noriega, J., Coelho, J., Neves, M., \& Martins, C. (2006). Valores humanos básicos como preditores do bem-estar subjetivo. Psico-USF, 37(2), 131-137.

Bardi, A., \& Goodwin., R. (2011). The dual route to value change: Individual processes and cultural moderators. Journal of Cross-Cultural Psychology, 42, 271-287. doi: 10.1177/0022022110396916

Belo, P., Gouveia, V., Raymundo, S., \& Marques, C. (2005). Correlatos valorativos do sexismo ambivalente. Psicologia: Reflexão e Crítica, 18, 7-15.

Brewer, M. B., \& Roccas, S. (2001). Individual values, social identity, and optimal distinctiveness. Em C. Sedikides \& M. B. Brewer (eds.), Individual Self, relational self, collective self (pp. 219-37). Ann Arbor, MI: Taylor \& Francis.

Brown, S., \& Lent, R. (2016). Vocational psychology: Agency, equity, and well-being. Annual Review of Psychology, 67, 27.1-27.25. doi: 10.1146/ annurev-psych-122414-033237

Chaves, S. (2003). Valores como preditores do bem-estar subjetivo (Dissertação de mestrado não publicada). Universidade Federal da Paraíba, João Pessoa, Brasil.

Coelho, J. M, Gouveia, V., \& Milfont, T. (2006). Valores humanos como explicadores de atitudes ambientais e intenção de comportamento pró-ambiental. Psicologia em Estudo, 11, 199-207.

Diener, E., \& Biswas, R. (2000). New directions in subjective well-being research: The cutting edge. Indian Journal of Clinical Psychology, 27(1), 21-33.

Diener, E., Suh, E. M., Lucas, R. E., \& Smith, H. L. (1999).Subjective well-being: Three decades of progress. Psychological Bulletin, 125(2), 276-302. doi: 10.1037//0033-2909.125.2.276

Fonseca, N., Chaves, S., \& Gouveia, V. (2006). Professores do ensino fundamental e bem-estar subjetivo: Uma explicação baseada em valores. Psico-USF, 11(1), 45-52.

Gouveia, V. (1998). La naturaleza de los valores descriptores del individualismo e del colectivismo: Una comparación intra e intercultural (Tese de doutorado não publicada). Universidade Complutense de Madrid, Madrid, Espanha.

Gouveia, V. (2003). A natureza motivacional dos valores humanos: Evidências acerca de uma nova tipologia. Estudos de Psicologia (Natal), 8, 431-443. doi: 10.1590/S1413-294X2003000300010.

Gouveia, V., Albuquerque, F., Clemente, M., \& Espinosa, P. (2002). Human values and social identities: A study in two collectivist cultures. International Journal of Psychology, 37, 333-342.

Gouveia, V., Barbosa, G., Andrade, E., \& Carneiro, M. (2005). Medindo a satisfação com a vida dos médicos no Brasil. Jornal Brasileira de Psiquiatria, 54(4), 298-305.

Gouveia, V., Milfont, T., \& Guerra, V. (2014). Functional theory of human values: Testing its content and structure hypotheses. Personality and Individual Differences, 60, 41-47. doi: 10.1016/j.paid.2013.12.012

Gouveia, V., Milfont, T., Fischer, R., \& Coelho, J. (2009). Teoria funcionalista dos valores humanos: Aplicações para organizações. S. Paulo. RAM - Revista de administração Mackenzie, 10(3), 34-59. doi: 10.1590/ S1678-69712009000300004

Gouveia, V., Santos, W., Milfont, T., Fischer, R., Clemente, M., \& Espinosa, P. (2010). Teoría funcionalista de los valores humanos en España: Comprobación de las hipótesis de contenido y estructura. Revista Interamericana de Psicologia/ Interamerican Journal of Psychology, 44(2), 203-214.

Gouveia, V., Veloso, V., Meira, M., Gusmão, E., Filho, M., \& Souza, L. (2008). Valores humanos e interesses vocacionais: Um estudo correlacional. Psicologia em Estudo, 13(3), 603-611. doi: 10.1590/ S1413-73722008000300022.

Gouveia, V., Vione, K., Milfont, T., \& Fischer, R. (2015). Patterns of value change during the life span some evidence from a functional approach to values. 
Personalityand Social PsychologyBulletin, 41, 1276-1290. doi: 10.1177/0146167215594189

Gusmão, E. S. (2004). A hipótese da congruência vocacional: Consideraçôes acerca dos valores bumanos e do bem-estar subjetivo (Dissertação de mestrado). João Pessoa. Departamento de Psicologia, Universidade Federal da Paraíba.

Hofstede, G. (1984). Culture's consequences: International differences in word-related values. Newbury Park, CA: Sage Publications.

Huta, V., \& Waterman, A. S. (2014). Eudaimonia and its distinction from hedonia: Developing a classification and terminology for understanding conceptual and operational definitions. Journal of Hapiness Studies, 15, 1425-1456. doi: 10.1007/ s10902-013-9485-0.

Inglehart, R. (1977). The silent revolution: Changing values and political styles among Western publics. Princeton, NJ: Princeton University Press.

Lent, R. W., Taveira, M.C., Sheu, H. B., \& Singley, D. (2009). Social cognitive predictors of academic adjustment and life satisfaction in Portuguese college students: A longitudinal analysis. Journal of Vocational Behavior, 74(2), 190-198. doi: 10.1016/j. jvb.2008.12.006.

Higgs, M., \& Dulewicz, V. (2014) Antecedents of wellbeing: A study to examine the extent to which personality and emotional intelligence contribute to well-being, The International Journal of Human Resource Management, 25(5), 718-735, doi:10.1080/095 85192.2013 .815253

Marques, C., Silva, A. D., Taveira, M. C., Mota, A., \& Gouveia, V. (2012, December). Basic values survey: Results of a confirmatory factor analysis with Portuguese youths living in foster care institutions. Poster session presented at the Hong Kong International Conference on Education, Psychology and Sciety. Hong Kong, China.

Medeiros, E. (2011). Teoria funcionalista dos valores bumanos: Testando sua adequação intra e interculturalmente (Tese de doutoramento não publicada). Departamento de Psicologia, Universidade Federal da Paraíba, João Pessoa, Paraíba.

Neto, F. (1993). The satisfaction with life scale: Psychometrics properties in an adolescent sample.
Journal of Youth and Adolescence, 22(2), 125-134. doi: 10.1007/BF01536648

Neto, F. (2001). Satisfaction with life among adolescents from immigrant families in Portugal. Journal of Youth and Adolescence, 30, 53-67.

Neto, F., Barros, J., \& Barros, A. (1990). Satisfação com a vida. Em L. Almeida, R. Santiago, P. Silva, L. Oliveira, O. Caetano, J. Marques \& J. Marques (Eds.). A acção educativa: Análise psico-social (pp. 91-100). Leiria, Portugal: ESEL/APPORT.

Nogueira, F. (2014). O espaço e o tempo da cidadania na educação. Revista Portuguesa de Pedagogia, 49(1), 7-32.

Porfeli, E. (2007). Work values system development during adolescence. Journal of Vocational Behaviour, 70(1), 42-60.

Pronk, S. (2010). Correlatos da imagem corporal: Uma explicação pautada na autoimagem e nos valores humanos (Dissertação de mestrado não publicada). Departamento de Psicologia, Universidade Federal da Paraíba, João Pessoa, Paraíba.

Pimentel, C. (2004). Valores humanos, preferência musical, identificação grupal e comportamento antissocial (Dissertação de mestrado não publicada). Departamento de Psicologia, Universidade Federal da Paraíba, João Pessoa, Paraíba.

Ramos, L. (2015). Os mecanismos sociocognitivos e o bem-estar psicologógico: Teste de um modelo integrativo em estudantes universitários (Tese de doutorado não publicada). Faculdade de ciências sociais e humanas, Universidade da Beira Interior, Covilha, Portugal.

Regulamento No 258/2011, Código Deontológico da Ordem dos Psicólogos Portugueses. Diário da República: $2^{a}$ série. Acedido em 27 de abril 2016. Reuperado de www.dre.pt

Rokeach, M. (1968).Beliefs, attitudes, and values. San Francisco: Jossey-Bass.

Rokeach, M. (1973). The nature of buman values. New York: Free Press.

Schwartz, S. (1992). Universals in the content and structure of values: Theoretical advances and empirical tests in 20 countries. Em M. Zanna (Eds.), Advances in experimental social psychology (pp. 1-65). New York: Academic Press. 
Schwartz, S. H. (2005). Robustness and fruitfulness of a theory of universals in individual human values. Em A. Tamayo \& J. B. Porto (Eds.), Valores e comportamento nas organizações (pp. 56-95). Petrópolis, Brazil: Vozes.

Sheldon, K. M. (2005). Positive value change during college: Normative trends and individual differences. Journal of Research in Personality, 39, 209-223.

Simões, A. (1992). Ulterior validação de uma escala de satisfação com a vida (SWLS). Revista Portuguesa de Pedagogia, 3, 503-515.
Tabachnick, B., \& Fidell, L. (2007). Using multivariate analysis. Needham Heights, Allyn e Bacon.

Vasconcelos, T. (2004). Personalidade, valores e condutas antissociais de jovens (Dissertação de mestrado não publicada). Departamento de Psicologia, Universidade Federal da Paraíba, João Pessoa, Paraíba.

Recebido em: $11 / 08 / 2015$

Reformulado em: 03/02/2016; 28/02/2016

Aceito em: 03/03/2016

Sobre as autoras:

Cátia Marques é doutorada em Psicologia Aplicada pela Universidade do Minho, bolsista de Investigação no Centro de Investigação em Psicologia da Escola de Psicologia da Universidade do Minho, com interesses de investigação no âmbito dos valores de vida, questionamento ético e gestão e desenvolvimento da carreira. E-mail: catiamarques@psi.uminho.pt

Ana Daniela Silva é doutorada em Psicologia pela Universidade do Minho, foi bolsista de investigação Pós-Doc nos últimos seis anos, no Centro de Investigação em Psicologia da Universidade do Minho, com o projeto de investigação "Percursos de Carreira de Jovens em Lares". Atualmente é presidente da Associação Portuguesa para o Desenvolvimento da Carreira, com interesses de investigação no âmbito da identidade, desenvolvimento da carreira e populações vulneráveis.

E-mail: anadanielasilva@sapo.pt

Maria do Céu Taveira é doutorada em Psicologia da Educação pela Universidade do Minho, docente e investigadora na Escola de Psicologia da Universidade do Minho. Atualmente é coordenadora da linha de investigação Desenvolvimento da Carreira e Aconselhamento no Centro de Investigação em Psicologia, com interesses de investigação no âmbito da exploração vocacional, gestão e desenvolvimento da carreira e avaliação das intervenções de carreira.

E-mail:ceuta@psi.uminho.pt

Contato com as autoras:

Maria do Céu Taveira

Escola de Psicologia, Universidade do Minho, Campus de Gualtar

Braga, Portugal

CEP: 4710-057

Psico-USF, Bragança Paulista, v. 22, n. 2, p. 207-215, mai./ago. 2017 
